\title{
Étude sur les systèmes d'échange des connaissances pour la santé des jeunes et la prévention des maladies chroniques : étude de cas menée dans trois provinces
}

\author{
D. Murnaghan, Ph. D. (1); W. Morrison, Ph. D. (2); E. J. Griffith, Ph. D. (3, 4); B. L. Bell, Ph. D. (1); \\ L. A. Duffley, B. Sc. (2); K. McGarry, M. Sc. (3); S. Manske, Ph. D. (5)
}

Cet article a fait l'objet d'une évaluation par les pairs.

\section{Résumé}

Introduction : Les équipes de recherche ont adopté un modèle d'étude de cas utilisant un cadre d'analyse commun dans le but d'étudier trois systèmes provinciaux (île-duPrince-Édouard, Nouveau-Brunswick et Manitoba) d'échange des connaissances. Ces trois systèmes visent à générer et utiliser des données probantes lors de l'élaboration des politiques, de la planification des programmes et des évaluations afin d'améliorer la santé des jeunes et de prévenir les maladies chroniques.

Méthodologie : Nous avons appliqué un modèle d'étude de cas pour examiner en profondeur les leçons apprises (c.-à-d. les principales conditions ou les principaux processus contribuant au développement de la capacité d'échange des connaissances) à l'aide d'une méthode de collecte de données multiples. Les activités de gestion, de synthèse et d'analyse des données ont été simultanées, itératives et continues. Les leçons apprises ont été classées en sept catégories.

Résultats : L'échange des connaissances est un processus complexe, qui exige des champions et des partenariats de collaboration, une adaptation aux divers intervenants et qui exige aussi que les régions soient préparées.

Analyse : Dans l'ensemble, les systèmes d'échange des connaissances peuvent accroître la capacité d'échange et d'utilisation des données probantes en allant au-delà de la collecte et de la transmission de données. Leurs aires d'influence sont l'établissement de nouveaux partenariats, des activités élargies d'échange des connaissances et le perfectionnement des approches axées sur les politiques et les pratiques liées à la santé des jeunes et à la prévention des maladies chroniques.

Mots-clés : échange des connaissances, santé des jeunes, prévention des maladies chroniques, utilisation des connaissances, données probantes à l'action, surveillance, partenariat

\section{Introduction}

Le fardeau des maladies chroniques est à la hausse à l'échelle mondiale, et $89 \%$ des décès au Canada ${ }^{1}$ leur sont attribuables.
Les jeunes Canadiens sont susceptibles de développer des maladies chroniques en raison de leurs taux élevés de comportements néfastes modifiables liés à la santé, tels que la sédentarité ${ }^{2,3}$, une mauvaise alimentation $^{4}$ et le tabagisme ${ }^{5}$ et, par conséquent, pourraient avoir une espérance de vie plus courte que celle de leurs parents $^{4}$. La réduction du risque la plus marquée pourrait être obtenue grâce à une intervention opportune tôt dans la vie ${ }^{6}$.

Cette croissance des taux de maladies chroniques nous incite à générer rapidement des données probantes pertinentes pour contribuer aux politiques et aux programmes liés à la santé des jeunes et pour les orienter. La planification fondée sur des données probantes améliore les programmes de prévention ${ }^{7,8}$ en favorisant le ciblage et l'évaluation des programmes et des politiques ainsi que l'établissement des priorités ${ }^{9}$. Par conséquent, des données contextuelles et pertinentes à l'échelle locale sur les facteurs de risque modifiables sont en demande.

Diverses expressions, notamment " échange des connaissances ", " application des connaissances » et " développement des connaissances ", font référence au processus qui consiste à réaliser une recherche dans le but d'appliquer efficacement les données qui en résultent. Selon la Fondation canadienne de la recherche sur les services de santé, l'échange des connaissances (EC) favorise l'interaction bidirectionnelle entre des groupes ayant des cultures distinctes, afin que les connaissances créées soient utiles et pertinentes pour tous les intervenants ${ }^{10,11}$. Cette définition est en accord avec l'approche philo-

Rattachement des auteurs :

1. Faculté de soins infirmiers, Université de l'île-du-Prince-Édouard, Charlottetown (Île-du-Prince-Édouard), Canada

2. Faculté d'éducation, Université du Nouveau-Brunswick, Fredericton (Nouveau-Brunswick), Canada

3. Épidémiologie et registre sur le cancer, Action Cancer Manitoba, Winnipeg (Manitoba), Canada

4. Département des sciences de la santé communautaire, Faculté de médecine, Université du Manitoba, Winnipeg (Manitoba), Canada

5. Propel Centre for Population Health Impact, Université de Waterloo, Waterloo (Ontario), Canada

Correspondance : Donna Murnaghan, Faculté de soins infirmiers, Université de l'Île-du-Prince-Édouard, 550, avenue University, Charlottetown (île-du-Prince-Édouard) C1A 4P3; tél. : 902-566-0749; téléc. : 902-566-0777; courriel : dmurnaghan@upei.ca 
sophique et les interventions proposées dans le cadre de cette étude.

Plusieurs cadres d'EC cernent les principaux processus, personnes et contextes nécessaires au développement des connaissances et à la prise de décision. Jacobson et collab. ${ }^{12}$ ont fourni un guide pratique visant à aider les chercheurs à recueillir l'information pertinente à propos des groupes cibles essentiels à l'EC. Les Instituts de recherche en santé du Canada définissent l'application des connaissances comme un processus dynamique et itératif qui englobe la synthèse, la diffusion, l'échange et l'application éthique des connaissances, ainsi que l'évaluation et la surveillance des activités de transfert des connaissances ${ }^{13}$. Un troisième cadre est le cadre de recherche de la transposition des connaissances à la pratique, qui est composé de deux cycles fluides, complexes et dynamiques : la création de connaissances et l'action ${ }^{11}$.

Bien que l'EC soit depuis longtemps reconnu comme essentiel à la transposition des connaissances à la pratique, la recherche visant à contribuer et à soutenir ce type d'activité demeure lacunaire. Au Canada, les intervenants des secteurs des politiques, de la pratique et de la recherche provenant d'organisations provinciales et nationales liées à la promotion de la santé et aux maladies chroniques s'entendent sur l'importance de mieux connaître les processus d'EC et les exemples de pratiques fondées sur des données probantes en contexte local, régional et provincial. Ils reconnaissent également le besoin d'adopter la pensée systémique en santé publique comme un moyen de traiter des enjeux complexes liés à la santé publique ${ }^{14}$.

Trois provinces ont créé de façon indépendante, à partir de cadres existants, leurs propres systèmes provinciaux d'EC sur la santé des jeunes : le School Health Action, Planning, and Evaluation System Prince Edward Island (SHAPES-PEI; http://www.upei.ca/cshr/shapes) à l'îledu-Prince-Édouard; le Sondage sur le mieux-être auprès des élèves et l'Initiative sur l'échange des connaissances au Nouveau-Brunswick (SWS/KE; http://www.unbf.ca/education/herg/sante/ index.php) et le Risk Factor Surveillance System au Manitoba (MRFSS; http://partners.healthincommon.ca). Chaque province a établi un processus de transposition des connaissances à la pratique reconnaissant l'importance des données probantes dans l'élaboration des mesures et l'importance de la prise en compte des mesures dans le perfectionnement des données probantes (voir figures 1, 2 et 3). Quatre composantes de base de l'EC liées à la santé des jeunes ont été définies dans les trois cadres provinciaux d'EC :

(1) Des systèmes de surveillance pour appuyer la planification et l'évaluation des politiques et des programmes à l'intention des enfants et des jeunes (c.-à-d. collecte de données locales, dont celles liées aux facteurs de risque);

(2) La capacité de résumer les données pertinentes liées aux types d'interventions qui se sont avérées efficaces (c.-à-d. interprétation de données obtenues par l'entremise de la littérature, des évaluations de programme et du contexte local);

\section{FIGURE 1}

\section{SHAPES - Modèle d'échange et de développement des connaissances de l'île-du-Prince-Édouard}

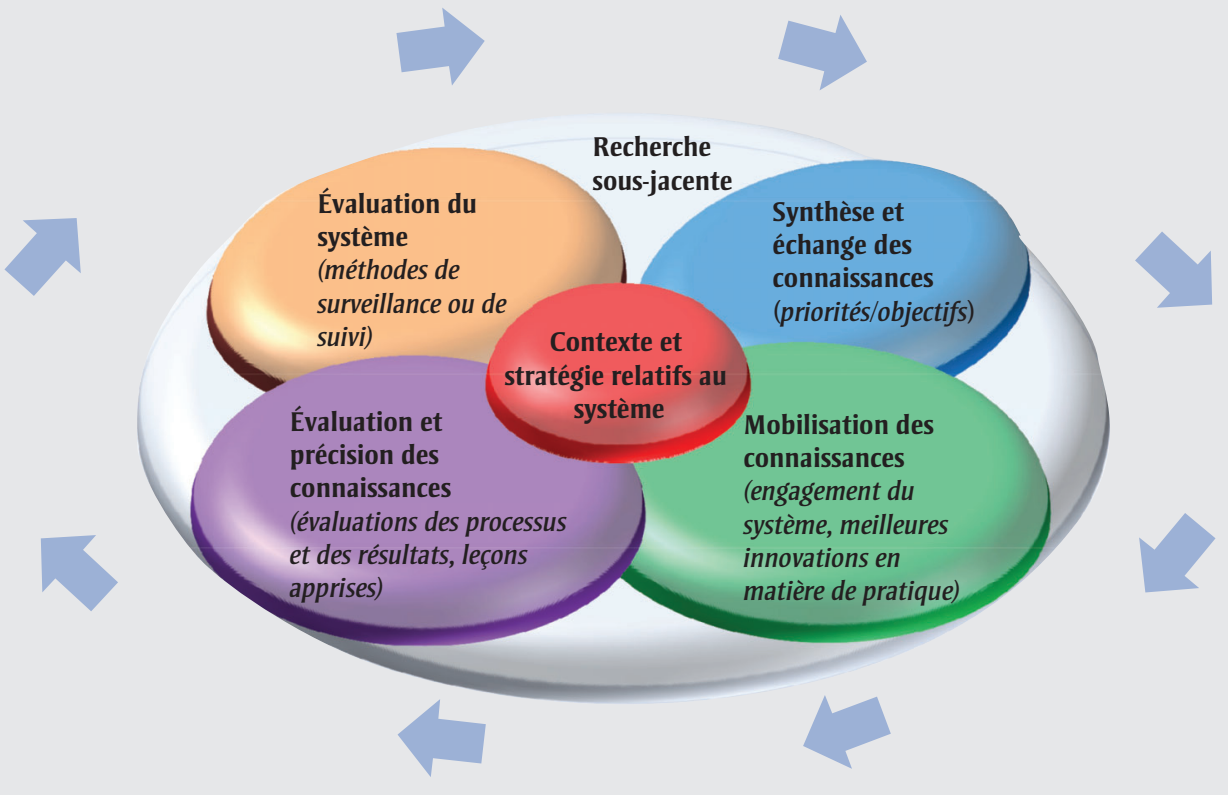

Abréviation : SHAPES, Système d'intervention, de planification et d'évaluation de la santé dans les écoles - Île-du-Prince-Édouard.

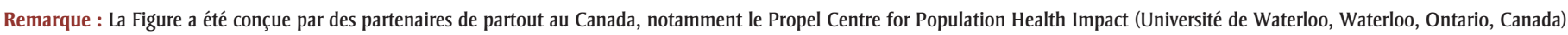
et le Groupe de recherche en santé et en éducation (Université du Nouveau-Brunswick, Fredericton, Nouveau-Brunswick, Canada). 
FIGURE 2

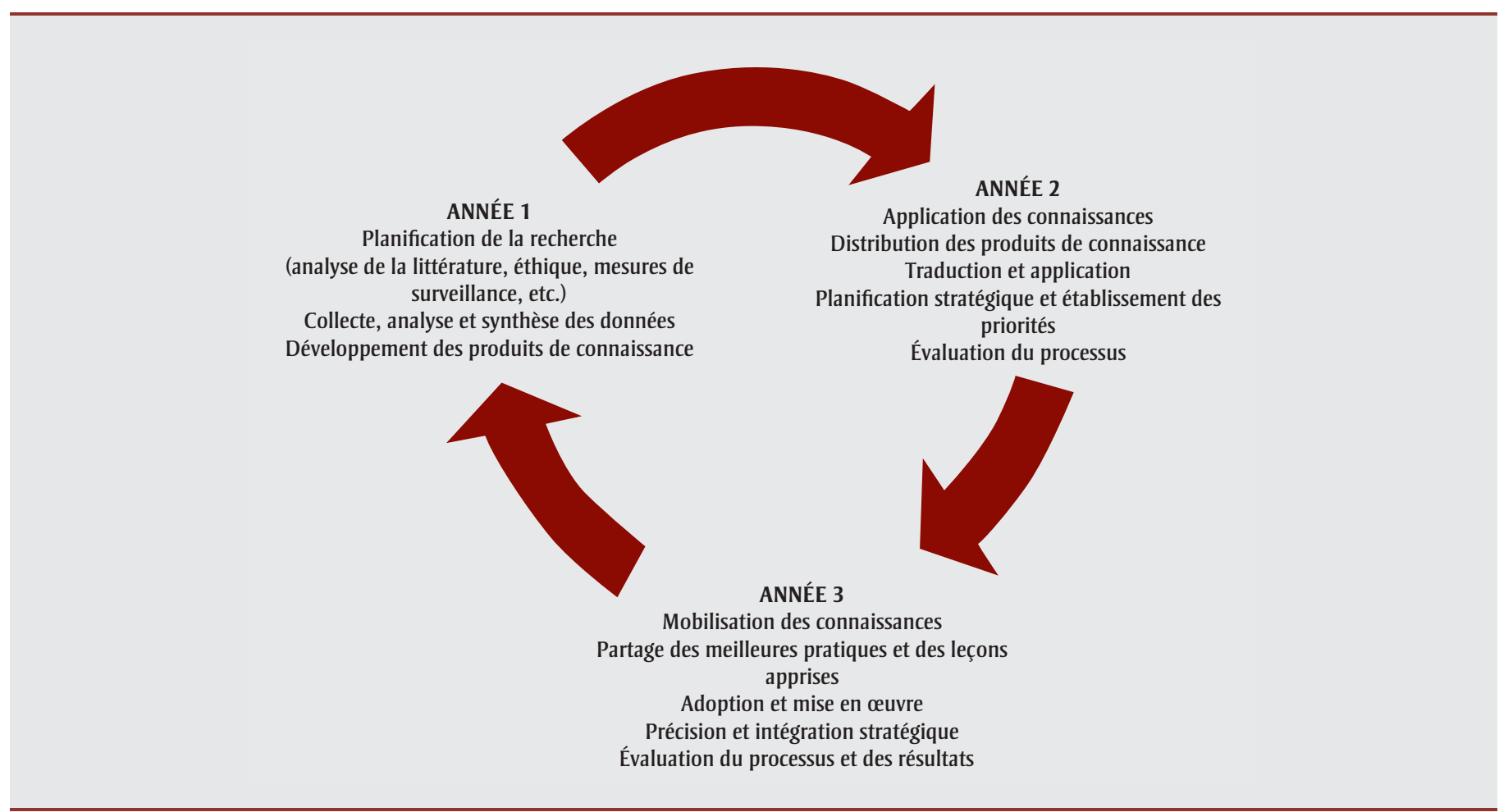

Remarque : Élaboré par des partenaires de partout au Canada, notamment le Groupe de recherche en santé et en éducation (Université du Nouveau-Brunswick, Fredericton, NouveauBrunswick, Canada) et le Propel Centre for Population Health Impact (Université de Waterloo, Waterloo, Ontario, Canada).

(3) La capacité de passer des données probantes à l'action (p. ex. utiliser les connaissances issues de l'interprétation des données pour mettre en œuvre de meilleures pratiques);

(4) Les moyens de générer des données probantes à partir des actions entreprises (c.-à-d. utiliser et partager les meilleurs programmes, pratiques, politiques, interventions, expériences et évaluations).

Cet article vise à présenter les leçons que l'on peut tirer de ces trois études de cas provinciales sur les systèmes d'EC liés à la santé des jeunes et à la prévention des maladies chroniques.

\section{Méthodologie}

Nous avons utilisé le modèle d'étude de cas Yin ${ }^{15}$ pour explorer le phénomène de l'EC liées à la santé des jeunes à l'échelle de trois provinces : le Manitoba, le Nouveau-Brunswick et l'Île-du-PrinceÉdouard. Le modèle d'étude de cas est utile pour répondre aux questions de types comment et pourquoi, tandis qu'on utilise plutôt un modèle d'étude de cas multiples pour explorer les différences entre les cas et au sein des cas, et prévoir des résultats similaires ou des résultats contradictoires, mais pour des motifs prévisibles ${ }^{15}$. Dans le cadre de cette étude, nous avons utilisé une méthode de collecte de données multiples pour mieux comprendre l'EC portant sur le contexte de vie réel de la santé des jeunes ${ }^{15}$.

\section{Procédure}

Les responsables de chaque étude de cas provinciale ont créé une équipe de recherche et un comité consultatif. De plus, les trois provinces ont créé une équipe de recherche multi-sites constituée des principaux chercheurs et du personnel de recherche de chaque province. Les protocoles d'étude ont servi à définir le but et l'orientation de la recherche, et chaque équipe provinciale a eu l'autonomie nécessaire pour explorer les cas à l'aide de la méthodologie la mieux adaptée à son contexte. Les équipes ont collaboré pour la mise au point des processus et des instruments de collecte de données. Les nombreuses sources de données probantes (analyses de documents, entrevues, groupes de discussion et même sondage en ligne pour l'île-du-PrinceÉdouard) ont amélioré la fiabilité et la validité des résultats de l'étude de cas (voir tableau 1$)^{15}$.

En collaboration avec les intervenants provinciaux et nationaux, les équipes de recherche ont élaboré des guides d'entrevue semi-dirigée (disponible sur demande). Les entrevues et les groupes de discussion ont été enregistrés puis transcrits et des notes d'observation sur le terrain ont aussi été rédigées après chaque entrevue $^{16}$. Les entrevues ont duré entre 45 et 60 minutes. Répondre au sondage en ligne structuré de l'Île-du-Prince-Édouard nécessitait entre 10 et 15 minutes. Ce dernier visait à comprendre les points de vue d'un plus grand éventail de partenaires, d'utilisateurs finaux et d'intervenants. Les documents examinés portaient sur la planification et les ressources et incluaient procès-verbaux de réunion, demandes de subvention, communications et coupures de presse. Des données ont été 
FIGURE 3

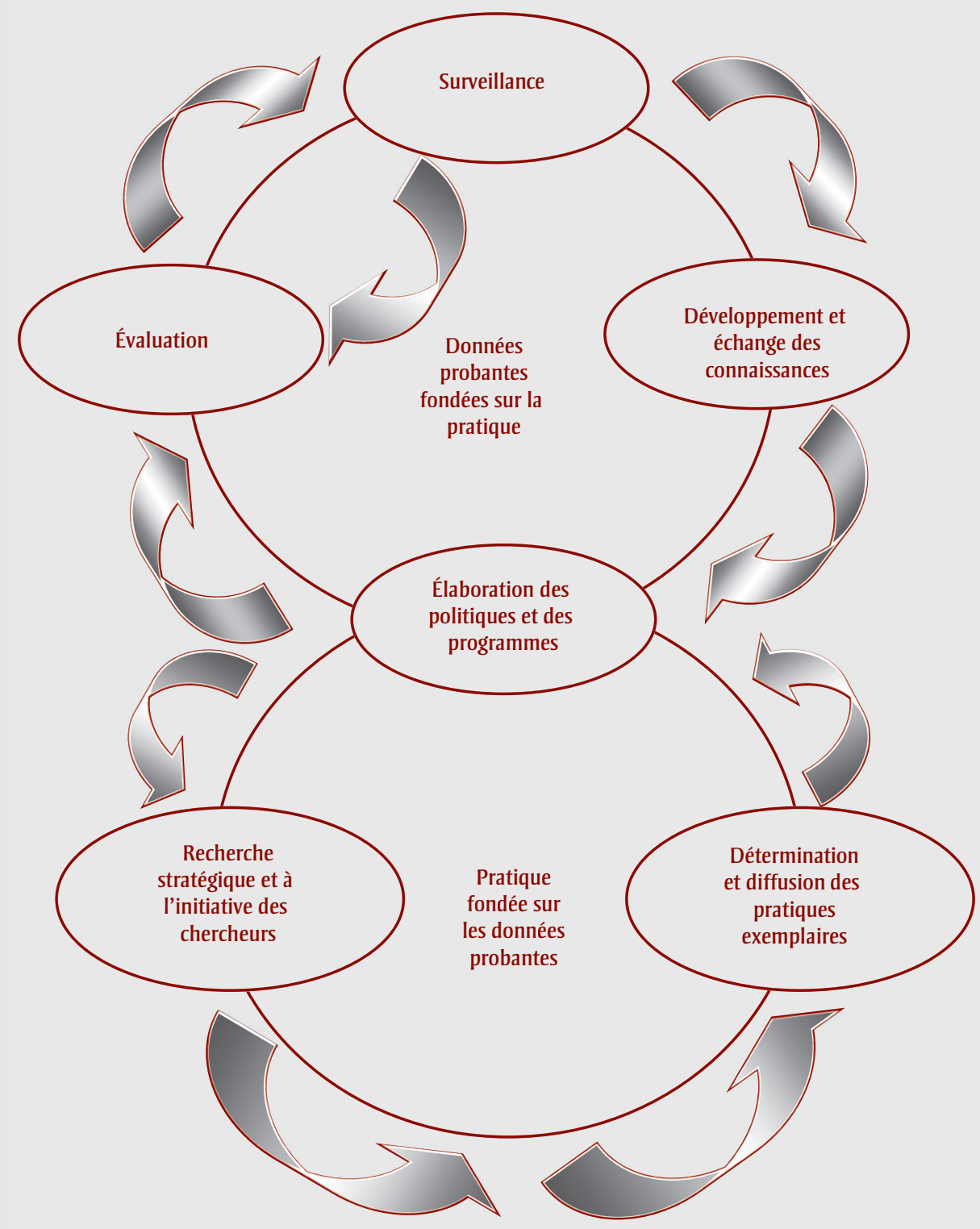

Source : Riley et Harvey, $2006^{18}$.

recueillies jusqu'à saturation (lorsque les thèmes cernés sont devenus répétitifs) pour chaque cas provincial.

Nous avons fait en sorte que les intervieweurs ne dirigent pas ou n'influencent pas les participants en partageant leurs opinions, etc. ${ }^{17}$ Les membres de l'équipe ont vérifié la saturation, se sont assurés que nous comprenions bien les thèmes émergents et que nos bilans témoignaient des contributions des participants, et ont clarifié et exploré en détail les entrevues initiales des participants. Environ six mois après les entrevues et les groupes de discussion initiaux, et après la fin des analyses préliminaires et la détermination des thèmes, nous avons partagé un premier bilan avec les participants. Cependant, seuls la moitié d'entre eux ont pu participer à ces entrevues de suivi.

Les comités d'éthique de chaque province ont approuvé cette recherche. 
TABLEAU 1

Résumé des activités de collecte de données

\begin{tabular}{lcccc} 
& Documents, $\mathbf{n}$ & Entrevues ${ }^{\mathbf{a}}, \mathbf{n}$ & $\begin{array}{c}\text { Répondants au } \\
\text { sondage, } \mathbf{n}\end{array}$ & $\begin{array}{c}\text { Groupes de discussion, } \\
\text { n (Participants, } \mathbf{n})\end{array}$ \\
\hline Man. & 137 & 32 & 0 & $6(35)$ \\
N.-B. & 78 & 32 & 0 & $2(48)$ \\
î.-P.-É. & 119 & 26 & 69 & $7(50)$ \\
\hline
\end{tabular}

Abréviations : Man., Manitoba; N.-B., Nouveau-Brunswick; Î.-P.-É., Île-du-Prince-Édouard.

${ }^{a}$ Nombre total d'entrevues réalisées (certains individus peuvent avoir participé à de multiples activités de collecte de données).

\section{Participants}

Nous avons utilisé un échantillonnage dirigé pour choisir les participants aux réseaux d'EC existants, puis un échantillonnage en boule de neige pour joindre les principaux intervenants. Tous les participants ont été informés du projet par courriel ou en personne, et ont donné leur consentement éclairé avant de participer. Les participants étaient des représentants des ministères provinciaux de la Santé ou du Mieux-être et de l'Éducation, des organisations non gouvernementales, des autorités sanitaires régionales, des écoles et des arrondissements scolaires, des universités et d'autres intervenants clés participant directement au système d'EC de leur province à titre de partenaires ou d'utilisateurs finaux (voir tableau 2). Moins de 10 participants de chaque province ont refusé de participer à l'étude.

\section{Analyse des données}

Les activités de gestion, de synthèse et d'analyse de données ont été simultanées,

itératives et continues. Nous avons utilisé le logiciel NVivo 8/9 (QSR International [Americas] Inc., Burlington, Massachussets, É.-U.) pour gérer et analyser les données. L’analyse a porté principalement sur les sondages thématiques et sur la description conceptuelle et thématique ${ }^{17}$. Chaque équipe provinciale a utilisé l'analyse thématique pour examiner et catégoriser les données de multiples sources et présenter ces données sous forme de tableaux. Les sous-ensembles de données ont été étiquetés et ordonnés par thèmes, et une analyse interprétative a été effectuée pour comprendre la signification des thèmes dégagés ${ }^{19}$. Les résultats ont été contre-vérifiés dans les rapports provinciaux définitifs, entre les participants, dans les analyses de documents et lors des discussions transversales.

Les équipes provinciales ont convenu d'utiliser une procédure d'analyse d'étude de cas multiples modifiée comme celle décrite par Stake ${ }^{20}$. Elles ont créé un cadre
TABLEAU 2

Descriptions des entrevues et des groupes de discussion

\begin{tabular}{lccc} 
Entrevues & î.-P.-É. $(\mathbf{n}=\mathbf{2 3})$ & N.-B. $(\mathbf{n}=\mathbf{3 2})$ & Man. $(\mathbf{n}=\mathbf{3 2})$ \\
\hline Rôles, \% & & & 0 \\
Recherche & 26 & 16 & 16 \\
Politique & 26 & 19 & 84 \\
Pratique & 39 & 65 & 0 \\
Autre & 9 & 0 & Man. $(\mathbf{n}=35)$ \\
\hline Groupes de discussion & î.-P.-É. $(\mathbf{n}=50)$ & N.-B. $(\mathbf{n}=\mathbf{4 8})$ & 0 \\
\hline Rôles, \% & & & 9 \\
Recherche & 0 & 8 & 91 \\
Politique & 0 & 15 & 0 \\
Pratique & 0 & 77 & 0 \\
Étudiant & 100 & 0 & \\
\hline
\end{tabular}

Abréviations : Man., Manitoba; N.-B., Nouveau-Brunswick; Î.-P.-É., Île-du-Prince-Édouard. initial à l'aide de thèmes déterminés à priori dans la littérature et de thèmes émergents découlant des discussions de cas dans chaque province et entre provinces. Leur résultats ont été classés en " groupes » prédéterminés. Ensuite, un processus itératif intensif à l'échelle des équipes provinciales a permis de définir les tendances présidant à l'élaboration du cadre définitif (voir tableau 3). Les stratégies, activités et partenaires communs ayant permis un meilleur EC ont été analysés en utilisant ce cadre.

Les résultats de cette étude transversale ciblent les similarités entre les systèmes d'EC, mais nous avons également recherché des contre-preuves afin d'éviter un biais holistique et de ne pas prêter une signification plus importante aux tendances que celle qui existe réellement ${ }^{17}$. L'examen des contre-preuves et des données probantes a entraîné modifications ou approfondissements du nouveau cadre. Le fait de cibler les similarités a fait émerger les éléments, processus et leçons apprises clés lors de la mise en œuvre d'un système d'EC. Ce processus à la fois inductif et déductif a permis d'approfondir la réflexion critique et de définir la gamme de l'impact potentiel des leçons à tirer de chaque cas provincial.

\section{Résultats}

Le contexte diversifié (social, politique, physique) de chaque système provincial d'EC a donné lieu à différents modèles de partenariat, de financement et de structure. Notre comparaison transversale a cependant permis de cerner des similarités entre les trois systèmes provinciaux d'EC, ce que nous considérons comme les leçons à tirer au sein des sept groupes thématiques définis. Il s'agit des conditions ou des processus clés contribuant au développement des capacités d'EC à l'échelle d'au moins deux contextes provinciaux. Des citations choisies de participants à la recherche ont été incluses afin de montrer l'importance de ces leçons apprises. Nous n'avons pas cité à dessein la province dans laquelle les entrevues particulières se sont déroulées, de manière à préserver l'anonymat de tous les participants. 
TABLEAU 3

Cadre analytique de comparaison transversale

\begin{tabular}{|c|c|c|}
\hline & Nom du groupe & Description du groupe \\
\hline 1. & Modèle directeur de l'échange des connaissances & $\begin{array}{l}\text { Cadres de systèmes existants dans lesquels sont définis les principaux } \\
\text { processus, intervenants et conditions contextuelles }\end{array}$ \\
\hline 2. & État de préparation & $\begin{array}{l}\text { Besoin reconnu en matière de données liées à la santé pour contribuer à l'élaboration } \\
\text { de politiques ou de pratiques aux échelons local, provincial ou national, et intérêt } \\
\text { manifeste des intervenants des domaines de la santé et du mieux-être et de l'éducation }\end{array}$ \\
\hline 3. & Produits d'échange des connaissances & $\begin{array}{l}\text { Ressources en matière de communication, telles que les rapports, les feuillets d'information, } \\
\text { les sites Web, visant à favoriser la participation de divers auditoires et à les informer }\end{array}$ \\
\hline 5. & $\begin{array}{l}\text { Partenariats stratégiques dans le domaine de l'échangé } \\
\text { des connaissances }\end{array}$ & Relations ou collaborations jouant un rôle de leadership ou d'influence clé \\
\hline 6. & Systèmes et structures & $\begin{array}{l}\text { Réseaux d'échangé des connaissances ou systèmes de prise de décisions nouveaux } \\
\text { ou émergents }\end{array}$ \\
\hline
\end{tabular}

Abréviation : EC, échange de connaissances.

\section{Modèles de base de l'échange des connaissances}

Les trois provinces ont utilisé des cadres de systèmes existants ainsi que les principaux processus, personnes et conditions contextuelles comme base de leurs initiatives de surveillance pour planifier et réaliser leurs activités, et orienter et communiquer les travaux en cours. Bien que ces modèles aient été différents dans chaque province, le recours à des modèles d'EC a aidé à communiquer et à comprendre les rôles des différents intervenants dans le développement, le partage ou l'application des connaissances. Deux personnes interviewées ont fourni ces explications :

Je crois que pour que le [sondage auprès des étudiants] remporte réellement un succès, les participants, qu'il s'agisse des directeurs d'école ou des parents ou des enfants [...] doivent avoir une idée de ce qui s'en vient et comprendre que l'information contribuera à la prochaine étape, et connaître le calendrier jusqu'à la prochaine étape, de manière à ce que tout le monde sache qu'il s'agit du début d'un processus plutôt que de la fin d'un processus. (province 1)
Il est essentiel qu'un plan et que l'établissement des priorités fassent partie de la façon dont nous menons nos activités. (province 2)

\section{2. État de préparation}

Les provinces ont reconnu la nécessité d'avoir accès à des données sur la santé pour contribuer à l'élaboration des politiques ou des pratiques, et les intervenants des domaines de la santé et du mieux-être et de l'éducation ont manifesté un intérêt pour la création d'activités d'EC liées à la santé des jeunes.

Certaines écoles sont complètement prêtes à se lancer dans de telles activités; d'autres écoles sont sur le point de participer. (province 1)

On manquait dans les trois provinces de données locales exhaustives sur les comportements liés à la santé des jeunes. Les réseaux, coalitions et relations de travail existants étaient essentiels pour offrir des bases solides à la promotion de la surveillance de la santé des jeunes et de l'EC pouvant contribuer à l'élaboration des politiques et des pratiques. Les champions faisant la promotion de l'établissement de la surveillance et des processus d'EC, et les facilitant, étaient issus de divers groupes d'intervenants.

Nous avons une région très diversifiée. Nous avons des populations et des secteurs affluents, en santé [...] ayant des taux élevés de maladies chroniques. La moyenne régionale nous positionne au milieu. Alors, avoir des données issues du milieu scolaire nous aiderait à réellement déterminer quels programmes doivent être mis en oeuvre dans quelles communautés. (province 3 )

\section{Produits d'échange des connaissances}

Les produits d'EC, par exemple les ressources en communication comme les rapports, les feuillets d'information, les sites Web, les bulletins d'information, les résumés de projet, les comptes rendus de conférence et les communications médiatiques utilisés pour mobiliser et informer divers auditoires ont fourni un point d'ancrage commun aux trois provinces pour lancer le dialogue avec les intervenants - nouveaux ou déjà établis. Plusieurs produits ont été utilisés pour présenter les bilans détaillés portant sur les comportements de santé des jeunes qui influent sur 
les maladies chroniques, tels qu'une saine alimentation, l'activité physique, le tabagisme et les aptitudes mentales. Des produits d'EC variés, rédigés dans un langage simple et clair, ont été conçus pour des auditoires et des groupes d'intervenants spécifiques (voir tableau 4). Des résumés ou des feuillets d'information concis présentant les principaux résultats ayant trait à la santé des jeunes ont été jugés attrayants et intéressants pour les principaux décideurs et chefs de file. Des sites Web ont été utilisés pour diffuser les données et les ressources sur la santé des jeunes dans un but d'EC auprès d'un plus grand nombre d'intervenants.

J'ai trouvé [le rapport sur le profil] facile à consulter et à lire, selon ma perspective. Je veux dire que je sais que certains parents pourraient avoir de la difficulté à le consulter, mais j'aime sa présentation [...] voici les données; voici ce qu'elles veulent dire; voici les mesures que vous pourriez prendre. (province 1)

Le site Web est fantastique. Pour les communautés isolées, il est très avantageux. Mon équipe le consulte assez souvent pour obtenir des ressources. (province 3)

\section{Activités d'échangée des connaissances}

Il était important pour chaque province de cibler les intervenants de tous les niveaux lors de l'échange d'information et il était essentiel de créer des activités d'EC favorisant la mobilisation - événements, tribunes, réunions, présentations ou séances de planification. Ces activités ont été planifiées et mises en œuvre en fonction des processus stratégiques de chaque modèle provincial d'EC. Les champions régionaux et provinciaux de l'EC ont souvent été choisis comme coordonnateurs, animateurs ou présentateurs des activités d'EC. Les activités d'EC ont été reconnues comme favorisant le rassemblement des intervenants et l'établissement de partenariats.

Ce sont ces occasions de partage et d'échange qui nous permettent d'établir de nouveaux réseaux et d'obtenir des idées et des succès [...] cela nous motive. (province 2)

Nous avons présenté l'information contenue dans les rapports et avons discuté de ce que cela voulait dire pour chacun. Ça leur a permis de poser des questions et nous a permis de clarifier les choses. (province 3)

\section{Partenariats stratégiques dans le contexte de l'échange des connaissances}

Le leadership et les collaborations établies entre les intervenants possédant une expertise en santé et en mieux-être des jeunes, en éducation et en recherche ont été reconnus comme essentiels pour appuyer et maintenir les initiatives de surveillance. L'établissement de partenariats au sein du secteur de l'éducation était fondamental pour l'obtention et le maintien de la participation des écoles et des arrondissements scolaires.

Sincèrement, nous avions consacré plusieurs années à bâtir ces relations nous avions fait les appels. Nous avions régulièrement des réunions avec eux. Nous nous sommes demandé ce que nous faisions de bien et de mal; ce que nous pouvions améliorer. Nous avons réellement travaillé fort pour y parvenir. (province 1)

Dans notre petite province, ce sont les praticiens qui nous permettent de réa- liser tant de choses avec si peu de ressources [...] Les partenariats sont la clé de la force de l'initiative. (province 2)

\section{Systèmes et structures}

Les réseaux et systèmes de prise de décision liés à l'EC, qu'ils soient nouveaux ou existants, ont été reconnus comme jouant un rôle clé dans le développement et l'expansion des capacités en matière d'EC. Les réseaux nationaux déjà établis ont fourni la structure initiale à partir de laquelle ont été amorcées et favorisées les relations entre les intervenants des domaines de la recherche, des politiques et de la pratique. Des coalitions, des groupes, des réseaux et des initiatives du domaine de la santé ont utilisé les données de surveillance de la santé des jeunes pour élaborer des programmes et promouvoir la santé. Les activités de surveillance et d'EC favorisaient l'établissement de comités et de structures de planification en matière de santé et de mieux-être des jeunes.

Le rôle [du réseau de santé en milieu scolaire] serait d'officialiser les discussions informelles et cela devrait être créé, car lorsque les joueurs changeront $[\ldots]$ ces conversations se poursuivront de manière officielle. (province 1)

Les membres tirent profit des contributions uniques de tous les partenaires en fonction de leurs expériences, de leurs ressources et de leur expertise. (province 3 )

\section{Résultats de l'échangé des connaissances}

Les intervenants ont obtenu de l'aide pour interpréter et utiliser les résultats de manière à pouvoir passer efficacement de la connaissance à l'action. Parmi les

TABLEAU 4

Produits d'échange des connaissances

\begin{tabular}{ll} 
Produit & \multicolumn{1}{c}{ Auditoire visé } \\
\hline Rapports scolaires et rapports sommaires & $\begin{array}{l}\text { Administrateurs scolaires, enseignants, élèves, parents, comités scolaires et communautaires } \\
\text { Rapports de district, de division et rapports sommaires }\end{array}$ \\
$\begin{array}{l}\text { Personnel des arrondissements scolaires et des divisions, conseils scolaires, communautés, } \\
\text { praticiens de la santé }\end{array}$ \\
Rapports régionaux & Praticiens de la santé, dirigeants municipaux \\
Rapports provinciaux et rapports sommaires & Ministères provinciaux, alliances en santé, organisations non gouvernementales, grand public
\end{tabular}


résultats de l'EC, notons l'application des résultats de la surveillance, l'évaluation des priorités, la mobilisation des partenaires et l'obtention de financement. Les programmes de subventions liés à la surveillance de la santé en milieu scolaire ont été associés à une prise en compte accrue des rapports sur l'EC et à l'utilisation de données probantes. Certains exemples de réussite ont constitué des sources importantes de motivation et d'apprentissage. La répétition des activités de surveillance et d'EC a fourni une base solide pour créer et maintenir des partenariats en matière de santé en milieu scolaire. L'utilisation, par les intervenants ministériels et les groupes externes, de données sur la santé des jeunes pour établir les plans et priorités régionaux et provinciaux en matière de santé et de mieux-être, ainsi que pour établir les points de repère des programmes, a été considérée comme contribuant de façon majeure au soutien des activités de surveillance et d'EC à l'échelle des écoles.

Certaines écoles ont intégré l'information issue du [sondage] aux plans d'amélioration continue des écoles. Cela fonctionne également à l'échelle des arrondissements. (province 2)

Je me souviens d'avoir reçu les résultats et, parce qu'il y avait la subvention relative aux modes de vie sains, nous l'avons partagée avec le conseil étudiant. Nous leur avons demandé comment ils voulaient utiliser les subventions, et de présenter une demande. (province 3)

\section{Analyse}

L'objectif de cet article est de présenter les leçons à tirer de l'élaboration et de la mise en œuvre de systèmes d'EC dans trois provinces canadiennes. Ces trois systèmes sont similaires. L'EC est un processus complexe qui exige des champions et des partenariats de collaboration, une adaptation de l'EC en fonction de divers intervenants et qui exige que les régions soient préparées. Tous ces éléments renforcent les capacités d'EC et les systèmes aboutissant à la création de résultats concrets dans la promotion de modes de vie sains.
Les résultats de notre étude transversale contribuent à la recherche empirique, encore limitée, portant sur les modèles d'EC. Les provinces ont soulevé des thèmes similaires, notamment la nécessité d'utiliser un modèle de base d'EC lors de la mise en œuvre de systèmes de ce type. Bien que les trois provinces aient adopté des approches spécifiques à leur contexte, les systèmes d'EC mis en œuvre étaient comparables, comme en témoigne le cadre analytique commun qui en est issu.

Plusieurs cadres décrivent des processus d'EC conçus spécifiquement pour combler l'écart entre le chercheur et l'utilisateur final, par exemple le cadre du processus de transposition des connaissances à l'action (Knowledge-to-Action Process Framework) ${ }^{11}$, le cadre contextuel fondé sur la compréhension des besoins des utilisateurs (Understanding-User Context Framework ${ }^{12}$ et le modèle d'application des connaissances (Model of Knowledge Translation $)^{13}$. Les trois modèles provinciaux d'EC leur sont similaires. Ils visent à inclure les intervenants dans les processus d'EC et reconnaissent le rôle du contexte dans le développement, l'interprétation et le transfert des connaissances. Les modèles provinciaux représentent l'aboutissement de nombreuses années d'efforts durant lesquelles des mesures ont été prises rapidement par les communautés motivées à utiliser des données probantes lors de leurs prises de décision. La répétition a permis aux politiques et aux pratiques d'être évaluées et perfectionnées. Lorsque des pratiques se sont avérées inefficaces, les systèmes ont été adaptés et ont intégré de nouvelles connaissances issues de systèmes appliquant efficacement des modèles d'utilisation des ressources et de renforcement des capacités. Lors de la répétition de l'application du modèle, la communication et la collaboration entre les partenaires ont été élargies, retravaillées et améliorées.

Le cadre analytique de l'EC de cette étude est fondé sur des données probantes issues de l'expérience « concrète » de trois administrations canadiennes, ce qui donne lieu à une meilleure compréhension de l’EC.

Il s'est révélé essentiel d'avoir des champions à tous les échelons (local, régional, provincial et national) pour favoriser la mise en œuvre et le maintien des activités de surveillance et d'EC. La mobilisation des réseaux et de ces champions a nécessité la promotion de l'importance de la prise de décision fondée sur des données probantes et des besoins de collecte et de compréhension des données locales. Conformément aux conclusions de Walter et collab. ${ }^{21}$, lorsque ces champions ont approuvé et utilisé des données sur la santé des jeunes pour élaborer des plans locaux, régionaux et provinciaux sur la santé et le mieux-être et pour établir les points clés des programmes, la valeur des activités locales de surveillance et d'EC s'est accrue pour tous les intervenants ${ }^{21}$. Les champions agissent comme catalyseurs lorsqu'ils présentent de nouvelles idées et pratiques, les approuvent ${ }^{21}$ et encadrent d'autres intervenants dans leurs prises de décision.

Les domaines de la recherche, des politiques et de la pratique ont souvent des priorités divergentes, utilisent un langage différent, respectent des échelles temporelles différentes et sont assujettis à différents systèmes de récompense $\mathrm{e}^{22,23}$. Les Centers for Disease Control and Prevention, par exemple, avaient besoin d'une conceptualisation et d'un langage communs afin d'approfondir leur compréhension du processus qu'ils avaient entrepris de transposition des connaissances en action $^{24}$. En établissant des partenariats de collaboration, les possibilités d'accroître la sensibilisation aux fonctions de travail et aux attentes liées aux partenariats favorisent la création d'un processus de compréhension réciproque qui, en retour, donne lieu au respect mutuel et à d'autres partenariats et mesures de collaboration. Les modèles et les cadres d'EC peuvent servir à la mobilisation de divers partenaires dans le cadre d'une approche systémique axée sur la prévention des maladies chroniques. Le recours à un modèle d'EC a aidé les intervenants à comprendre et à maintenir leur participation à des activités de transposition des connaissances à la pratique liées à la santé des jeunes, et à s'y investir.

De plus, des partenariats de travail positifs au sein du secteur de l'éducation ont été essentiels pour obtenir et maintenir la participation des écoles et des arrondissements scolaires. En maintenant des rela- 
tions positives par l'entremise d'un processus réciproque et respectueux clairement articulé, tous les partenaires ont été encouragés à contribuer et se sont sentis valorisés. Gagnon ${ }^{25}$ a cerné quatre facteurs essentiels pour intégrer avec succès l'EC aux réseaux et aux communautés de pratique : l'établissement d'une compréhension partagée du problème de santé; la description explicite des rôles et des responsabilités de chacun; des compétences et des expériences au sein des membres de l'équipe permettant d'établir et de maintenir des collaborations efficaces et une stratégie de maintien des relations.

Les principales mesures de collaboration entre les provinces étaient la planification commune des approches de surveillance et le choix du moment, et ont porté sur la manière dont les données seraient utilisées et partagées par les administrations locales, régionales et provinciales. La génération conjointe de connaissances influerait sur l'adoption et l'utilisation des travaux de recherche, en permettant une meilleure prise en compte des facteurs contextuels et une capacité accrue de les traiter, créant ainsi une information crédible et valide utile pour les intervenants et en laquelle ils ont confiance ${ }^{26}$. Les connaissances portant sur des secteurs d'intérêt et des priorités intéressant les intervenants accroissent la probabilité qu'elles soient utilisées ou appliquées ${ }^{27,28}$. Williams et collab. ${ }^{29}$ ont souligné l'importance de favoriser la participation des utilisateurs finaux à toutes les activités clés qui témoignent du processus de développement des connaissances. Toutefois, les exemples de partenariats de collaboration soutenus et de communication continue entre les producteurs et les utilisateurs finaux des connaissances sont rares et inhabituels ${ }^{30}$. Notre recherche montre que la répétition des activités de surveillance et d'EC a aidé à soutenir les partenariats dans le domaine de l'EC sur la santé des jeunes. Ces partenariats ont évolué et se sont élargis lors des activités communes de surveillance et d'EC.

La mobilisation de chefs de file de divers groupes d'intervenants a renforcé les capacités à prendre des mesures préliminaires liées aux activités de surveillance et d'EC d'envergure provinciale. Les succès remportés grâce aux activités de surveillance liées à la santé et à la planification du transfert des connaissances à l'action ont favorisé, tant de la part des individus que de celle des organisations, un plus grand engagement et un soutien accru pour la surveillance de la santé des jeunes, tout comme l'obtention de données probantes à partir des mesures prises. Ward et collab. ${ }^{31}$ ont également établi que les caractéristiques et les contextes personnels, interpersonnels, organisationnels et professionnels ont une incidence sur les processus d'EC, ce qui confirme l'importance de tabler sur les atouts disponibles, tels que l'expertise, les partenariats et l'infrastructure, lors de la mise en œuvre d'un système d'EC ${ }^{31}$.

Les échanges de collaboration sont facilités lorsque les résultats des EC pertinents sont consultés et utilisés. Nous avons mis en évidence l'importance d'adapter les produits d'EC aux divers groupes d'intervenants. Les facettes intéressantes de ces produits étaient l'utilisation d'un langage simple et d'information pertinente sur le plan local, l'inclusion d'exemples des meilleures pratiques, l'intégration de données probantes fondées sur la pratique ou d'exemples de réussite, et la disponibilité de rapports, de résumés ou de feuillets d'information en divers formats et lieux. Les produits d'EC, tels que les feuillets d'information, les sites Web, les bulletins d'information, les rapports, les résumés de projet, les comptes rendus de conférence et les communications médiatiques, ont favorisé la collaboration entre les chercheurs et les utilisateurs de la recherche $\mathrm{e}^{24,32}$. Les produits d'EC devraient inclure des suggestions de mesures favorisant davantage l'application et l'utilisation des données ${ }^{33,34}$.

Des activités d'EC variées sont essentielles pour toucher et intéresser divers intervenants : consultations individuelles avec les intervenants sur les résultats et les meilleures pratiques en matière de santé et de mieux-être des jeunes, présentations de groupe sur les résultats en milieu scolaire dans les arrondissements scolaires et les provinces, événements fondés sur les conclusions locales et régionales concernant la surveillance, et enfin présentations de documents officiels dans le cadre de conférences. Des réunions en personne, officielles et non officielles, réunissant des chercheurs, des décideurs et des praticiens s'avèrent la façon la plus efficace de surmonter le manque d'échange entre partenaires $^{25}$. De plus, ces activités d'EC ont lieu dans un cadre élargi qui permet l'interaction entre de nombreux partenaires ayant des priorités, des processus, des contextes, des attentes et des incitatifs au changement dynamiques. Par conséquent, le recours à des stratégies d'EC qui offrent un choix suffisant aux utilisateurs finaux en matière de contenu, de format et de présentation s'est avéré important dans un contexte d'application et d'utilisation des données probantes ${ }^{27}$.

Étant donné l'accroissement des taux de maladies chroniques, il est urgent que le Canada génère et utilise des données probantes pertinentes pour contribuer à des interventions et à des politiques et programmes efficaces à l'intention des jeunes et portant sur des modes de vie sains. Des travaux de recherche ont montré que la planification fondée sur des données probantes améliore les programmes de prévention des maladies chroniques $^{7,8}$ lorsqu'elle est utilisée pour cibler et évaluer les programmes et les politiques et établir les priorités ${ }^{9}$. La génération ponctuelle de données n'est pas suffisante pour évaluer les programmes et les politiques visant les maladies chroniques et pour surveiller les changements relatifs à la santé des jeunes. L'utilisation de la pensée systémique peut réduire l'écart entre la génération, la dissémination et l'utilisation des données ${ }^{14}$. La pensée systémique est un outil clé de l'intégration de la production et de l'utilisation des connaissances pertinente pour les interventions locales ${ }^{14}$.

\section{Limites}

Nos conclusions peuvent être appliquées à d'autres administrations partageant des caractéristiques similaires à celles du Manitoba, du Nouveau-Brunswick et de l'Île-du-Prince-Édouard. Des travaux de recherche devraient s'intéresser à l'application de nos conclusions sur ces trois provinces principalement rurales à des administrations plus importantes et urbaines dans le cadre de situations 
complexes. Les études sur les interventions devraient porter sur divers produits et activités d'EC afin de vérifier leur efficacité. Il faudrait également avoir des outils et des modèles de partenariats plus précis pour faciliter les processus d'EC axés sur la santé des jeunes. Bien que ce soit surtout les similarités entre les trois systèmes d'EC qui aient été décrites ici, des différences ont été constatées entre les systèmes. Elles n’ont pas été décrites en détail, mais plutôt cernées dans le cadre de notre analyse afin d'éliminer le biais holistique.

\section{Conclusion}

Nos conclusions appuient une approche systémique des EC accroissant les capacités d'échange et d'utilisation des données probantes allant au-delà de la simple collecte de données et de la production de rapports. Ces systèmes peuvent contribuer à l'expansion de l'établissement de partenariats et d'activités de partage des connaissances, ainsi qu'à la création d'initiatives stratégiques et pratiques globales conçues pour promouvoir la santé des jeunes et la prévention des maladies chroniques.

\section{Remerciements}

Cette étude a été financée grâce à une contribution financière de Santé Canada, par l'entremise du Partenariat canadien contre le cancer. Les opinions exprimées sont celles des auteurs et ne représentent pas nécessairement les opinions du bailleur de fonds. Les auteurs aimeraient également remercier Courtney Laurence, les partenaires de l'initiative COALITION Youth Excel, les membres de l'équipe de l'étude de cas de chaque province et tous les participants à la recherche.

\section{Références}

1. Organisation mondiale de la Santé. Prévention des maladies chroniques : un investissement vital [Internet]. Genève $(\mathrm{CH})$, Organisation mondiale de la Santé; 2005 [consultation le 24 février 2012]. PDF téléchargeable à partir de la page : http:// www.who.int/chp/chronic_disease_report /contents/en/index.html
2. Jeunes en forme Canada. Ne laissons pas ces quelques pas être la source la plus importante d'activité physique de nos enfants après l'école. Bulletin de l'activité physique chez les jeunes de 2011 de Jeunes en forme Canada. [Internet]. Toronto (Ont.) : Jeunes en forme Canada; 2011 [consultation le 24 février 2012]. PDF (8,52 Mo) téléchargeable à partir du lien : http:// dvqdas9jty7g6.cloudfront.net/reportcard2011 /ahkc2011_shortform_fr_final.pdf

3. Freeman JG, King M, Pickett W et collab. La santé des jeunes Canadiens : un accent sur la santé mentale. Ottawa (Ont.), Agence de la santé publique du Canada; 2011. [Agence de la santé publique du Canada, $n^{\circ}$ de catalogue : 978-1-100-98001-0].

4. Marshall H, Boyd R. L'administrateur en chef de la santé publique : Rapport sur l'état de la santé publique au Canada [Internet]. Ottawa (Ont.), Agence de la santé publique du Canada; 2008 [consultation le 24 février 2012]. [Santé publique du Canada, n de catalogue : HP2-10/2008F]. PDF (3,97 Mo) téléchargeable à partir du lien : http://www.phac-aspc.gc.ca/cphorsphc -respcacsp/2008/fr-rc/pdf/CPHO-Report-f.pdf

5. Santé Canada. Préoccupations liées à la santé : Sommaire des résultats de l'Enquête sur le tabagisme chez les jeunes 2008-2009 [Internet]. Ottawa (Ont.); [modification le 20 août 2010; consultation le 27 février 2012]. Consultable en ligne à la page : http://www.hc-sc.gc.ca/hc-ps/tobac-tabac /research-recherche/stat/_survey-sondage _2008-2009/result-fra.php

6. Hanson M, Gluckman P. Developmental origins of noncommunicable disease: population and public health implications. Am J Clin Nutr. 2011;94(6 supplemental): 1754S-8S.

7. Brownson RC, Smith CA, Jorge NE, Deprima LT, Dean CG, Cates RW. The role of data-driven planning and coalition development in preventing cardiovascular disease. Public Health Rep. 1992 Jan-Feb; 107(1):32-7.

8. Alciati $\mathrm{MH}$, Glanz K. Using data to plan public health programs: experience from state cancer prevention and control programs. Public Health Rep. 1996;111(2): 165-72.
9. Mokdad AH, Remington PL. Measuring health behaviors in populations. Prev Chronic Dis. 2010;7(4):A75.

10. Canadian Health Services Research Foundation. Glossary of knowledge exchange terms used by CHSRF [Internet]. Ottawa (ON): CHSRF; [consultation le 18 septembre 2012]. Consultable en ligne à la page : http://74.81.206.232/Publications AndResources/ResourcesForResearchers /KEYS/GlossaryOfKnowledgeExchangeTerms .aspx

11. Graham ID, Logan J, Harrison MB et collab. Lost in knowledge translation: time for a map? J Contin Educ Health Prof. 2006;26: 13-24.

12. Jacobson N, Butterill D, Goering P. Development of a framework for knowledge translation: understanding user context. J Health Serv Res Policy. 2003;8(2): 94-9.

13. Instituts de recherche en santé du Canada. À propos de l'application des connaissances aux IRSC - L'application des connaissances : définition [Internet]. Ottawa (Ont.), IRSC; [consultation le 19 septembre 2012]. Consultable en ligne à la page : http://www.cihr-irsc.gc.ca/f/39033.html

14. Best A, Moor G, Holmes B et collab. Health promotion dissemination and systems thinking: towards integrative model. Am J Health Behav. 2003;27(suppl 3):S206-16.

15. Yin RK. Case study research: design and methods. 4th ed. Thousand Oaks (CA): Sage Publications; 2009.

16. Halcomb EJ, Davidson PM. Is verbatim transcription of interview data always necessary? Appl Nurs Res. 2006;19(1): $38-42$.

17. Miles MB, Huberman AM. Qualitative data analysis: a sourcebook of new methods. Thousand Oaks (CA): Sage Publications; 1984.

18. Riley B, Harvey D. A Manitoba Integrated Knowledge System: for the Primary Prevention of Chronic Disease [Internet]. Manitoba (MB): Partners in Planning for Healthy Living; [consultation le 5 avril 2013]. PDF (240 Ko) téléchargeable à partir du lien : http://www.healthincommon.ca /wp-content/uploads/Manitoba-Integrated -Knowledge-System-Feb-2006.pdf 
19. Sandelowski M, Barroso J. Classifying the findings in qualitative studies. Qual Health Res. 2003 Sept;13(7):905-23.

20. Stake RE. Multiple case study analysis. New York: The Guilford Press; 200.

21. Walter I, Nutley S, Davies H. What works to promote evidence-based practice? A cross-sector review. Evid Policy. 2005;1(3): 335-64.

22. Rynes SL, Bartunek JM, Daft RL. Across the great divide: knowledge creation and transfer between practitioners and academics. Acad Manage J. 2001;44(2):340-55.

23. Jansen MW, van Oers HA, Kok G. et NK. de Vries. Public health: disconnections between policy, practice and research [Internet]. Health Res Policy Syst. 2010 [consultation le 22 mars 2012];8(37). Consultable en ligne à la page : http:// www.health-policy-systems.com/content $/ 8 / 1 / 37$

24. Wilson KM, Brady TJ, Lesesne C, on behalf of the NCCDPHP Work Group on Translation. An organizing framework for translation in public health: the knowledge to action framework. Prev Chronic Dis [Internet]. 2011 [consultation le 22 mars 2012];8(2). Consultable en ligne à la page : http://www.cdc.gov/pcd/issues/2011/mar /10_0012.htm

25. Gagnon ML. Moving knowledge to action through dissemination and exchange. J Clin Epidemiol. 2011 Jan;64(1):25-31.

26. Lomas J. Decision support: a new approach to making the best healthcare management and policy choices. Healthc Q. 2007;10(3): 16-8.

27. Dobbins M, DeCorby K, Twiddy T. A knowledge transfer strategy for public health decision makers. Worldviews Evid Based Nurs. 2004;1(2):120-8.

28. Rosenbaum P. From research to clinical practice: considerations in moving research into people's hands. Personal reflections that may be useful to others. Pediatr Rehabil. 2005;8(3):165-71.

29. Williams A, Holden B, Krebs P et collab. Knowledge translation strategies in a community-university partnership: examining local Quality of Life (QoL). Soc Indic Res. 2008;85(1):111-25.
30. Broner N, Franczak M, Dye C, McAllister $\mathrm{W}$. Knowledge transfer, policymaking and community empowerment: a consensus model approach for providing public mental health and substance abuse services. Psychiatr Q. 2001;72(1):79-102.

31. Ward V, Smith S, House A, Hamer S. Exploring knowledge exchange: a useful framework for practice and policy. Soc Sci Med. 2012 Feb;74(3):297-304.

32. Kiefer L, Frank J, Di Ruggiero E et collab. Fostering evidence-based decision-making in Canada: examining the need for a Canadian population and public health evidence centre and research network. Can J Public Health. 2005; 96(3):I1-40.

33. Dobbins M, Hanna SE, Ciliska D et collab. A randomized controlled trial evaluating the impact of knowledge translation and exchange strategies. Implement Sci. 2009;4:61.

34. Lavis J, Robertson D, Woodside J et collab. How can research organizations more effectively transfer research knowledge to decision makers? Milbank Q. 2003; 81(2):221-48. 